\title{
UTILIZAÇÃO DE DIRETRIZES CLÍNICAS EM CARDIOLOGIA NA SAÚDE SUPLEMENTAR NO BRASIL
}

\author{
Claudia Caminha Escostegur*, Margareth Crisóstomo Portela, Sheyla Maria lemos lima, Vanja Maria Bessa Ferreira, Maurício Teixeira leite de \\ VASCONCELLOS, CLÁUDIA BRITO \\ Trabalho realizado pela Escola Nacional de Saúde Pública, Fundação Oswaldo Cruz, Rio de Janeiro, RJ
}

\author{
${ }^{*}$ Correspondência: \\ Av. Alexandre Ferreira 361 - \\ Lagoa \\ CEP 22470-220 - Rio de \\ Janeiro-RJ \\ Tel: (21) 2527-4553; Fax: \\ (21) 2535-3802 \\ cescosteguy@hse.ri.saude.gov.br
}

\begin{abstract}
RESUMO
OBjetivo. Apresentar resultados da área cardiovascular de um estudo nacional sobre a utilização de diretrizes clínicas na saúde suplementar.

Métodos. Estudo transversal, baseado em amostra representativa das 1573 operadoras de planos de saúde do Brasil, estratificada por macrorregião e segmento do mercado, subdividida em estratos certos e amostrados pelo método de Hedlin, com seleção eqüiprovável feita pelo algoritmo de Hájek em cada estrato amostrado, resultando em 90 operadoras entrevistadas com questionário estruturado; estimação pontual de porcentagens, totais e médias através de fatores de expansão, relativas a uma população $\mathrm{N}=1572$.

Resultados. Para 61,2\% das operadoras a utilização de diretrizes clínicas deve ser conduzida por órgãos reguladores governamentais, com participação de operadoras, prestadores de saúde e associações médicas. Apenas 32,3\% das operadoras conduzem a utilizaçãa de diretrizes clínicas, variando de 6,5\% (filantrópicas) a 38,2\% (autogestão). Entre as operadoras que conduzem o uso de diretrizes, a área cardiovascular apresenta uma das maiores utilizações: infarto agudo do miocárdio (87\%); insuficiência cardíaca (85\%); procedimentos invasivos em cardiologia (81,1\%); hipertensão arterial $(74,1 \%)$; acidente vascular encefálico $(72,2 \%)$; diabetes $(65,4 \%)$. As sociedades médicas são a fonte mais comum dessas diretrizes. $64,1 \%$ das operadoras monitoram indicadores de processo/resultado dos prestadores, mas apenas 10,5\% monitoram o uso de reperfusão coronariana no IAM de forma sistemática. 73,2\% promovem campanhas de promoção/ prevenção de agravos: hipertensão arterial (97\%), diabetes (93,3\%), obesidade $(70,7 \%)$ e tabagismo (60,8\%).

Conclusä̃o. Implementação baixa e incipiente de diretrizes, com variação regional e por segmentos do mercado. De uma forma geral, a área cardiovascular apresentou uma das maiores utilizações de diretrizes.

UnITERMOS: Diretrizes. Diretrizes para a prática clínica. Diretrizes clínicas. Saúde suplementar. Doença cardiovascular. Cardiologia.
\end{abstract}

\section{INTRODUÇÃO}

As diretrizes clínicas (DC) vêm sendo internacionalmente utilizadas como instrumento da gestão da clínica, constituindo-se em posicionamentos ou recomendações sistematicamente desenvolvidos para orientar médicos e pacientes acerca de cuidados de saúde apropriados, em circunstâncias clínicas específicas'. Proliferaram-se a partir da década de 90, como resposta à crescente preocupação com o estímulo ao uso e efetiva utilização de práticas endossadas pelo conhecimento científico corrente, tendo como motivação a melhoria da qualidade da assistência, mas também, de forma progressiva, a alocação mais eficiente de recursos ${ }^{2}$. Hoje, consolidam-se sob o pressuposto amplamente aceito de que sua implementação, com base na evidência científica disponível acerca da eficácia e efetividade de intervenções, produz melhores resultados na populaçãa assistida. ${ }^{3,4}$ Entretanto, a utilização de diretrizes varia mundialmente, em geral não sendo elevada devido à baixa adesão pelos profissionais de saúde e pelos pacientes ${ }^{2,5-7}$.
As doenças cardiovasculares exercem um papel preponderante na morbimortalidade mundial. No Brasil, constituem-se na principal causa de mortalidade e têm impacto relevante na carga de doença total do país ${ }^{10,11}$. Por outro lado, trata-se de uma área com grande disponibilidade de ensaios clínicos randomizados, na qual têm havido, internacionalmente, grandes esforços para o desenvolvimento de DC baseadas em evidência científica. Há assim, na atenção às doenças cardiovasculares, uma sensibilização relativamente diferenciada para o desenvolvimento e uso de DC, mas também, em diversos países, incluindo o Brasil, grande variação na adesão a essas diretrizes, marcada tanto pela presença da subutilização de tecnologias recomendadas como pela superutilização de tecnologias inadequadas segundo o conhecimento científico existente ${ }^{12-19}$.

Recentemente foi realizado um estudo nacional sobre a percepção de gestores de operadoras de planos de saúde sobre a utilização de DC na saúde suplementar. O objetivo desse estudo foi caracterizar o uso de $D C$ e outros instrumentos e práticas de gestão da clínica entre 
operadoras de planos de saúde no Brasil, permitindo a sistematização dos tipos, fontes e nível de fundamentação em evidência científica dessas diretrizes e a identificação dos desafios e estratégias para a sua implementação ${ }^{7}$. Esse estudo nasceu de uma demanda da Agência Nacional de Saúde Suplementar (ANS) com a Organização PanAmericana de Saúde (OPAS), e estimou que em apenas 32,3\% das operadoras de planos de saúde no país os gestores relataram o uso de DC, havendo variação entre regiões do país e entre segmentos do mercado. Este artigo é parte do referido estudo nacional sobre a utilização de DC na saúde suplementar, e tem o objetivo de caracterizar a utilização de DC na área cardiovascular.

\section{Métodos}

O estudo foi transversal, baseado na realização de um inquérito nacional em amostra representativa do conjunto de 1.573 operadoras de planos de saúde do Brasil, que constavam do cadastro de seleção fornecido pela ANS e atualizado até 18/08/2004. Por razões orçamentárias, o tamanho da amostra foi fixado em 100 operadoras e, para assegurar representatividade nacional, a amostra foi, inicialmente, estratificada por macrorregião e segmento do mercado. Diante da forte assimetria da distribuição dos beneficiários, observada na maior parte dos estratos iniciais da amostra, o método de otimização proposto por Hedlin ${ }^{20}$ foi para estratificar os estratos iniciais, de forma a assegurar a seleção da amostra mais eficiente dentre as possíveis amostras de tamanho 100.

Este tamanho foi alocado entre os estratos iniciais de forma proporcional ao seu número de beneficiários, sendo a distribuição do tamanho entre estrato certo e amostrado, de cada estrato inicial, o resultado da aplicação do método de Hedlin, programado para minimizar a variância do estimador de total do número de beneficiários (única variável quantitativa do cadastro recebido). Ajustes no tamanho de amostra decorrentes dos arredondamentos, da necessidade de assegurar a seleção de pelo menos duas operadoras por estrato amostrado, e do tamanho dos estratos previamente considerados como certos (Quatro operadoras), aumentaram o tamanho da amostra para I I 2 operadoras. Nos estratos amostrados foi usado o algoritmo de Hájek ${ }^{21}$ para selecionar uma amostra aleatória simples de operadoras.

Durante o trabalho de campo, alguns problemas foram enfrentados levando à necessidade de substituição das operadoras inicialmente selecionadas. A equipe de pesquisadores contatou 164 operadoras, das quais 25 apresentavam erro de cadastro (operadora não encontrada, comprada ou fusão com outra, falida, etc.), 55 recusaram-se a prestar informações e as 90 restantes foram entrevistadas, constituindo a amostra efetivamente trabalhada $(80,4 \%$ de resposta em relação ao tamanho ajustado de I I 2 operadoras). Essa amostra, resultante de 90 operadoras, expandida corresponde a I.572 das 1.573 operadoras da população contemplada, assim distribuídas: 326 autogestões (20,7\%) com 9.369.64I beneficiários (18,8\%); 370 cooperativas médicas (23,5\%) com I I .983.86 I beneficiários (24\%); I 24 filantrópicas (7,9\%) com 2.305.457 beneficiários (4,6\%); 739 medicina de grupo (47\%) com 18.4 I 8.752 beneficiários (36,9\%); e I 3 seguradoras (0,8\%) com 7.771 .200 beneficiários (I5,6\%). Uma das seguradoras recusou-se a prestar informações e não pôde ser substituída porque era única no estrato (mais detalhes da amostra em outra publicaçãō).

A coleta de dados entre as operadoras, realizada entre novembro de 2004 a março de 2006, foi feita através de um questionário estruturado, focalizando variáveis relativas ao seu conhecimento acerca de DC, bem como motivações, objetos e finalidades envolvidos no uso de DC. O questionário foi aplicado em entrevista pessoal, após assinatura do termo de consentimento informado, buscando-se a interlocução do responsável pela área da assistência, geralmente o diretor médico. Os principais componentes do questionário envolveram um diagnóstico do nível de sensibilização e implementação de operadoras no que concerne às DC, identificando áreas mais focalizadas entre diretrizes existentes, grau de difusão e acompanhamento do uso de diretrizes, perspectivas e resultados do uso de diretrizes relativos a custos e qualidade da assistência, e principais dificuldades na implementaçãao de diretrizes. Também se buscou identificar o uso de outros instrumentos e práticas de gestão assistencial, incluindo, critérios de credenciamento de prestadores de serviços, monitoramento de indicadores e realização de pesquisas de satisfação dos usuários.

Os dados coletados foram armazenados em um banco criado no ACCESS ${ }^{\circledR}$. A análise foi descritiva, através do pacote estatístico $S A S^{\circledR}$, levando-se em conta um fator de expansão da amostra atribuído por estrato final da amostra, para a obtenção das estimativas sobre o universo de operadoras de planos de saúde.

\section{Resultados}

No geral, 507 operadoras de planos de saúde (32,3\%) declararam conduzir o uso de diretrizes clínicas, havendo grande variação segundo segmentos do mercado. Os maiores percentuais de utilização declarada foram os da autogestão (38,2\%, correspondendo a 125 operadoras), medicina de grupo (37,7\% ou 278 operadoras) e cooperativas médicas ( $25,8 \%$ ou 95 operadoras); os menores foram os das seguradoras $(7,7 \%$, correspondendo a apenas uma operadora) e entidades filantrópicas (6,5\%, ou oito operadoras). Também variou por regiões do país, havendo um maior relato de uso na região Sudeste (43,4\%), seguido do Centro-Oeste (35,4\%), Sul (22,5\%), praticamente inexistente no Nordeste $(0,5 \%)$ e não tendo sido relatado na região Norte.

Entre as operadoras que reportaram utilizar diretrizes clínicas $(n=507)$, as áreas com maiores relatos de aplicação de diretrizes foram: assistência pré-natal (91,8\%), infecção hospitalar (91,2\%), infarto agudo do miocárdio (87\%), insuficiência cardíaca (85\%), câncer de mama ( $81,9 \%)$, câncer de colo uterino $(81,9 \%)$, uso de procedimentos invasivos em cardiologia $(81,1 \%)$, câncer de próstata $(79,6 \%)$ e hipertensão arterial $(74,1 \%)$.

De uma forma geral, considerando-se a atenção cardiovascular, a utilização relatada de diretrizes foi alta para todos os agravos analisados. A Tabela I identifica as áreas de utilização em cardiologia - incluindo diabetes - de acordo com o segmento do mercado; os percentuais referem-se ao total de operadoras que reportaram a condução da implementaçãao de DC. Observou-se grande variação dessa utilização de acordo com o segmento do mercado. A análise das filantrópicas e das seguradoras é limitada pelo número reduzido de operadoras que 
Tabela I - Estimativa do uso de diretrizes clínicas em cardiologia entre as 507 operadoras de planos de saúde que reportaram conduzir a sua utilização, segundo segmento do mercado

\begin{tabular}{|c|c|c|c|c|c|c|c|c|c|c|c|c|}
\hline \multirow[t]{2}{*}{ Diretrizes clínicas } & \multicolumn{2}{|c|}{ Total } & \multicolumn{2}{|c|}{ Autogestão } & \multicolumn{2}{|c|}{ Cooperativa } & \multicolumn{2}{|c|}{ Filantrópica } & \multicolumn{2}{|c|}{ Medicina de grupo } & \multicolumn{2}{|c|}{ Seguradora } \\
\hline & $\mathrm{n}$ & $\%^{(1)}$ & $\mathrm{n}$ & $\%^{(1)}$ & $\mathrm{n}$ & $\%^{(1)}$ & $\mathrm{n}$ & $\%^{(1)}$ & $\mathrm{n}$ & $\%(1)$ & $\mathrm{n}$ & $\%^{(1)}$ \\
\hline Infarto agudo do miocárdio & $44 \mid$ & 87,0 & 25 & 100,0 & 76 & 80,0 & 8 & 100,0 & 232 & 83,5 & 0 & 0,0 \\
\hline Insuficiência cardíaca & 431 & 85,0 & 23 & 98,4 & 69 & 72,6 & 8 & 100,0 & 231 & 83,1 & 0 & 0,0 \\
\hline Procedimentos invasivos em cardiologia & 411 & 81,1 & 24 & 99,2 & 94 & 98,9 & 8 & 100,0 & 184 & 66,2 & । & 100,0 \\
\hline Hipertensãoarterial & 376 & 74,1 & 88 & 70,4 & 94 & 98,9 & 7 & 87,5 & 187 & 67,3 & 0 & 0,0 \\
\hline Acidente vascular encefálico & 366 & 72,2 & 24 & 99,2 & 49 & 51,6 & 8 & 100,0 & 185 & 66,5 & 0 & 0,0 \\
\hline Diabetes & 332 & 65,4 & 87 & 69,6 & 51 & 53,7 & 7 & 87,5 & 187 & 67,3 & 0 & 0,0 \\
\hline
\end{tabular}

(I) As porcentagens referem-se ao total de operadoras que responderam SIM ao uso na área específica em relação ao total de operadoras que reportaram a condução do uso (totais na Figura I).

Tabela 2 - Base, forma e realização de avaliação da adesão no uso de diretrizes clínicas em cardiologia entre operadoras de planos de saúde que reportaram conduzir a sua utilização $(n=507)$

\begin{tabular}{|c|c|c|c|c|c|c|c|}
\hline \multirow[t]{2}{*}{ Diretrizes clínicas } & \multirow{2}{*}{$\frac{\text { Total que conduz }}{n}$} & \multicolumn{2}{|c|}{ Baseada em evidência } & \multicolumn{2}{|c|}{ Uso na forma original } & \multicolumn{2}{|c|}{ Avaliação da adesãc } \\
\hline & & $n$ & $\%^{(1)}$ & $n$ & $\% \%^{(1)}$ & $n$ & $\%^{(1)}$ \\
\hline Infarto agudo do miocárdio & $44 \mid$ & 422 & 95,7 & 170 & 38,4 & 223 & 66,8 \\
\hline Insuficiência cardíaca & 431 & 412 & 95,6 & 187 & 43,3 & 215 & 64,4 \\
\hline Procedimentos invasivos em cardiologia & 4|| & 392 & 95,4 & 142 & 34,4 & 193 & 57,9 \\
\hline Hipertensão arterial & 376 & 357 & 94,9 & 150 & 39,9 & 195 & 58,5 \\
\hline Acidente vascular encefálico & 366 & 319 & 94,4 & $|4|$ & 38,5 & 149 & 44,6 \\
\hline Diabetes & 332 & 313 & 94,3 & 148 & 44,7 & 197 & 59,0 \\
\hline
\end{tabular}

(I) As porcentagens referem-se ao total de operadoras que responderam SIM em relação ao total de operadoras que responderam ao quesito.

reportaram o uso de DC (apenas oito filantrópicas e uma seguradora); no caso da única seguradora, o uso limitou-se à área de procedimentos invasivos. Considerando-se os demais segmentos, de uma forma geral, a autogestão apresentou os melhores percentuais de utilização, destacando-se, particularmente, com relação ao infarto agudo do miocárdio (IAM), procedimentos invasivos em cardiologia, acidente vascular encefálico (AVE) e insuficiência cardíaca. A utilização relatada para hipertensão arterial e para diabetes foi menor do que nas demais áreas.

A Tabela 2 identifica áreas, formas e avaliação da adesão das diretrizes entre as operadoras que reportaram conduzir sua utilização, na área cardiovascular. Apesar do percentual de utilização de diretrizes ter variado bastante entre os agravos considerados (Tabela I), uma vez utilizadas, o relato de que eram baseadas em evidência científica foi consistentemente elevado para todas as áreas (acima de 94\% em todos os casos). Já o uso das diretrizes, como originalmente concebidas, foi reduzido (abaixo de 45\% em todos os casos). A avaliação da adesão dos prestadores de serviço às diretrizes foi maior no caso do IAM e menor no AVE.

A Figura I apresenta as fontes relatadas segundo os agravos considerados, podendo haver mais de uma fonte por agravo. As sociedades médicas constituíram a fonte predominante. Para procedimentos invasivos em cardiologia, o peso das sociedades médicas foi relativamente menor, havendo também uma participação importante do consenso de especialistas e das próprias operadoras.

O desenvolvimento de políticas de incentivo ao uso de diretrizes foi relatado por $64,2 \%$ das operadoras que reportaram o uso de

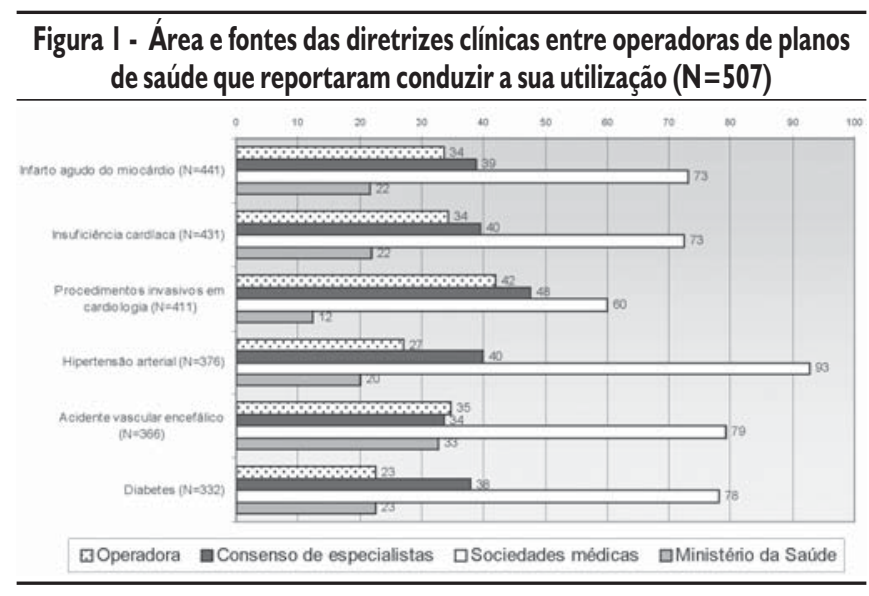

diretrizes. A freqüência desse desenvolvimento variou de acordo com o agravo: para doença cardíaca foi 55,2\%, hipertensão arterial (55\%), diabetes ( 45,7\%) e doença cerebrovascular (35,8\%).

Considerando-se aquelas operadoras que relataram conduzir o uso de diretrizes na área do IAM, apenas 21,3\% referiram orientar o uso de reperfusão coronariana de forma sistemática, sendo a maioria dessas, cooperativas médicas.

No geral, 73,2\% das operadoras referiram promover campanhas de promoção/prevenção em diversas áreas, destacando-se em especial as condições de risco para as doenças cardiovasculares: hipertensão 
arterial, 71\%; diabetes, 68,3\%; obesidade, 51,8\%; tabagismo, 44,5\%; alcoolismo, 33,5\%. Esses percentuais variaram muito segundo o segmento do mercado. De uma forma geral, foram menores para as seguradoras.

Os dirigentes das operadoras entrevistados se expressaram livremente sobre as principais dificuldades e estratégias identificadas para a implementação de DC no âmbito da saúde suplementar. Considerando o conjunto de operadoras, as dificuldades mais citadas referiramse à resistência por parte dos prestadores (médicos e hospitais), à inadequação da estrutura interna das operadoras e às características constitutivas da operadora; à inadequação das estratégias e práticas utilizadas pelos órgãos reguladores e de classe para estimular e assegurar o uso de diretrizes - identificação inadequada dos agravos e condições para os quais as diretrizes devem ser construídas, mecanismos e formatos inadequados de construção e divulgação de diretrizes, insuficiência e fragilidade das iniciativas e desarticulação entre os órgãos reguladores, inclusive com o poder judiciário; a baixa adesão dos beneficiários; a não valorização na formação médica do uso das diretrizes clínicas; e a natureza do problema e do trabalho em saúde.

Ainda considerando o conjunto de operadoras, foram propostas as seguintes estratégias: ações que visem maior adesão, comprometimento e conscientização, especialmente de prestadores, mas também de usuários, com destaque para a divulgação da importância do uso de DC junto aos prestadores, para melhoria da qualidade e para a racionalização dos custos e na busca de consenso entre os diferentes atores envolvidos no processo; maior estruturação interna das operadoras para que, efetivamente, possam utilizar e monitorar o uso de diretrizes junto aos prestadores; definição e instituição de políticas e normas mais claras, objetivas e incisivas pelos órgãos reguladores e formuladores de políticas na esfera estatal; e readequação da formação médica, enfatizando o enfoque dos custos e da variabilidade da prática médica.

\section{Discussão}

Ao caracterizar o conjunto de operadoras de planos de saúde no país, ressalta-se a predominância da medicina de grupo e o fato do segmento das seguradoras ter um peso relevante no número de beneficiários, embora represente o menor percentual de operadoras. Há uma grande concentração de operadoras na região Sudeste $(59,5 \%)$ em contraposição às demais regiões - Sul (17,0\%), Nordeste (I3,0\%), Centro-Oeste $(7,2 \%)$ e Norte $(3,3 \%)^{7}$.

Além de limitado em termos quantitativos (32,3\%), o uso de DC entre operadoras de planos de saúde também carece do respaldo de políticas bem estruturadas para a sua efetiva implementação. Para $88 \%$ das operadoras, a condução do uso de DC deve envolver uma parceria entre órgãos reguladores tais como MS, ANS e Secretarias de Saúde, a participação de operadoras, prestadoras de serviços de saúde e associações profissionais. A condução feita por órgãos reguladores governamentais com a participação das operadoras, dos prestadores e das associações profissionais foi apontada por $61,2 \%$ das operadoras como sendo o processo mais adequado, enquanto que $27 \%$ das operadoras preferem que a condução seja feita pela operadora em conjunto com os seus prestadores. Entre as operadoras que referiram a condução da implementação de DC, a motivação mais freqüentemente reportada e priorizada foi a melhoria da qualidade de assistência, seguida pelo aumento da eficiência na alocação dos recursos?

De uma forma geral, o estudo nacional evidenciou uma incorporaçãa maior de diretrizes nos campos da atenção cardiovascular e oncológica, coincidindo com a tendência internacional2,7. O uso mais intenso de diretrizes nos campos da atenção cardiovascular é coerente com o perfil de morbimortalidade do país ${ }^{10,11}$. O estudo Carga de Doença no Brasil demonstrou, em seus resultados, que as doenças crônico-degenerativas responderam por $66,3 \%$ da carga de doença no país ${ }^{10}$.

A fonte mais utilizada de DC em todas as áreas é a das sociedades médicas, embora haja variação no uso, em termos de fontes e de utilização em formato original, de acordo com a área de atenção. A experiência brasileira de desenvolvimento e implementação de DC remonta ao Projeto Diretrizes e a algumas iniciativas isoladas de Sociedades Médicas, entre as quais se destaca a Sociedade Brasileira de Cardiologia (SBC). O Projeto Diretrizes, iniciado em outubro de 2000, é coordenado pela Associação Médica Brasileira (AMB) e o Conselho Federal de Medicina (CFM), constituindo um grande esforço no sentido da elaboração de diretrizes médicas, baseadas nas evidências científicas disponíveis, com vistas a "auxiliar na decisão médica e otimizar o cuidado aos pacientes" 2,22. A área cardiovascular conta com DC para dislipidemia, hipertensão arterial e tromboembolismo venoso. Por outro lado, a Sociedade Brasileira de Cardiologia tem desempenhado um papel muito atuante em relação a DC em cardiologia no Brasil, com publicações acerca de DC e consensos sobre diversos agravos desde 1998, todos disponibilizados na sua página e revista ${ }^{23}$.

Esses esforços certamente precisam ser compartilhados pelo Ministério da Saúde, ANS, Agência Nacional de Vigilância Sanitária (Anvisa), agências de fomento à pesquisa e instituições acadêmicas. É desejável que tal esforço leve em consideração a grande experiência e produção internacional, bem como a evidência científica provida pelos estudos realizados. Entretanto, ainda assim, demandará a realização de avaliação tecnológica e pesquisa clínica no país, com vistas à identificação de especificidades locais. Por outro lado, deverá estar ajustado às determinações da Anvisa, responsável pela regulação formal pertinente à aprovação de medicamentos, equipamentos e outras tecnologias, e contar com o endosso das Sociedades Médicas, que efetivamente poderão legitimar as diretrizes propostas junto à comunidade médica².

A condução da utilização de DC na saúde suplementar ainda é bastante incipiente em termos de difusão, de incentivos, de monitoramento e de avaliação de resultados. Surpreendentemente, 14,3\% das operadoras que reportaram a condução do uso de DC não difundem as diretrizes entre seus prestadores de serviço e $44,4 \%$ utilizam o mecanismo da justificativa de glosa como forma de difusão. Trinta e quatro por cento das operadoras que conduzem o uso de diretrizes não fazem nenhum tipo de avaliação formal da adesão dos prestadores às diretrizes. A forma de avaliação mais utilizada é o acompanhamento de pedidos de exames, medicamentos ou procedimentos, seguida pela avaliação de prontuários. No caso de solicitação de procedimentos que não constam das diretrizes, a análise do médico auditor é a conduta mais utilizada? 
A prática de gestão da clínica ainda é muito incipiente entre operadoras de planos de saúde no Brasil. Ainda que a melhoria da qualidade da assistência e o aumento da eficiência na alocação de recursos tenham sido apontados como as principais motivações para o uso de DC, observou-se que os indicadores assistenciais mais utilizados entre todas as operadoras de saúde foram o tempo médio de permanência e a taxa de exames por consulta ambulatorial. ${ }^{7}$ Mesmo entre as operadoras que declararam conduzir diretrizes na atenção ao IAM, apenas $21,3 \%$ orientam e avaliam o uso da reperfusão coronariana, o que na literatura internacional é considerado um indicador de qualidade de assistência ao IAM24.

No geral, 65,8\% das operadoras que reportaram a utilização de diretrizes referiram implementar a avaliação da adesão dos prestadores de serviço às diretrizes. A avaliação dessa adesão variou muito de acordo com o agravo. Na área cardiovascular, essa avaliação foi mais expressiva para o IAM $(66,8 \%)$ e insuficiência cardíaca $(64,4 \%)$ do que para hipertensão arterial (58,5\%), procedimentos invasivos $(57,9 \%)$ e $\operatorname{AVE}(4,6 \%)$.

Algumas limitações do estudo devem ser discutidas. A principal refere-se à coleta de dados a partir da informação dos dirigentes; não foi possível a mensuração da efetiva utilização das diretrizes, por razões operacionais. Sendo assim, os resultados estão sujeitos a viés de informação. É possível que o viés superestime a utilização de diretrizes, que ainda assim foi muito limitada. Há ainda a possibilidade de presença de viés de seleção, em função do elevado número de recusas de participação por operadoras, considerando o pressuposto de que possivelmente quem recusa apresenta piores condições. Entretanto, o desenho da amostra garante a representatividade do estudo.

Em termos gerais, esta pesquisa é estatisticamente representativa da realidade nacional, refletindo, entretanto, eventuais problemas do cadastro utilizado como base para a definição da amostra e a possibilidade de presença de viés de seleção. Apesar dos limites, ela propiciou a apreciação de que o uso de DC e outras práticas da gestão assistencial/clínica é ainda muito limitado e incipiente entre operadoras de planos de saúde atuantes no mercado de saúde suplementar do país. Ainda que seja pertinente considerar a hipótese de favorecimento a respostas "politicamente corretas", a estratégia e o instrumento de coleta de dados também permitiram a captação de vários aspectos que balizaram tais respostas. Nesse sentido, destacam-se, por exemplo, a contradição entre a elevada referência ao uso de diretrizes baseadas em evidência científica e a predominância de diretrizes adaptadas, bem como a ênfase na melhoria da qualidade assistencial como critério prioritário de motivação para o uso de diretrizes, quando as escassas práticas de avaliação da prática médica vigente centram-se em estratégias com foco nos custos. Esse fato ocorreu não apenas relacionado à área da atenção cardiovascular, mas também nas demais área estudadas. Outra contradição está no fato de a quase totalidade dos entrevistados ter considerado relevante a implementação de diretrizes clínicas mas apenas 32,4\% relataram conduzir sua utilização ${ }^{7}$.

Ainda que com índices médios predominantemente baixos, há importante variação no uso de DC e outras práticas de gestão clínica tanto geográfica quanto em termos dos segmentos do mercado de saúde suplementar. Especificamente, chamam a atenção os resultados referentes às seguradoras, que certamente expressam uma postura assumida de desvinculação do seu papel da assistência à saúde e os resultados relativos ao segmento filantrópico, que exceto para o uso corrente de DC, foi positivamente diferenciado para vários aspectos. Esses últimos, ainda que suscitem dúvidas, talvez sejam parcialmente explicados pela estreita vinculação das operadoras filantrópicas aos hospitais mantenedores.

Vale ainda sublinhar a necessidade de se avançar no processo de regulação da assistência não apenas cardiológica mas à saúde como um todo, envolvendo os diferentes atores que devem compartilhar tal processo. As iniciativas observadas em operadoras ainda estão muito centradas em serviços ou pessoas, carecendo de serem, de fato, incorporadas como políticas de direção e, talvez, mais que isso, políticas do sistema de saúde, com amplo compromisso com a qualidade dos cuidados de saúde oferecidos. Neste sentido, o Pacto pela Saúde 2006 talvez seja o primeiro documento geral do MS que prioriza a adoção de diretrizes como política de saúde pelos municípios, mencionando, inclusive, as necessidades de: definição de critérios para inclusão e exclusão de medicamentos e CID na Tabela de Procedimentos, com base nos protocolos clínicos e nas diretrizes terapêuticas; auditoria assistencial ou clínica amparada em procedimentos, protocolos e instruções de trabalho normatizados e pactuados; e adoção de protocolos clínicos e diretrizes terapêuticas, em consonância com os protocolos e diretrizes nacionais e estaduais ${ }^{25}$.

\section{Conclusão}

Segundo o relato dos dirigentes, a implementação de $D C$ na saúde suplementar é ainda baixa e incipiente, com variação regional e por segmentos do mercado. De uma forma geral, a área cardiovascular apresentou uma das maiores utilizações de diretrizes, com destaque para o infarto agudo do miocárdio, a insuficiência cardíaca e os procedimentos invasivos. O papel das sociedades de especialidades como fonte das diretrizes foi enfatizado.

\section{Conflito de interesse: não há}

\section{SUMMARY}

\section{Utilization of clinical guidelines by health PLAN operators IN the Brazilian health system}

BACKGROUND. The objective of this study is to present cardiovascular results of a national study about the implementation of clinical guidelines and other instruments and practices for clinical care management among health plan operators in Brasil.

METHODS. This was a cross-sectional study based on a representative sample of the 1,573 Brazilian health plan operators, stratified by macro region and market segment. Each stratum was subdivided in take-all and take-some strata by Hedlin's method, with equiprobability selection in each take-some stratum. The resulting sample size was of 90 health plan operators who were interviewed using a structured questionnaire. The study had a descriptive nature and, to obtain estimates for percents and total number of health plan operators relative to a population size of $N=1572$., sample weights were taken into account. 
RESULTS. Of the health plan operators, $61.2 \%$ considered that the implementation of clinical guidelines should be conducted by administrative and regulatory government organizations, involving a partnership with health plan operators, health service providers and medical societies. It was shown that only $32.3 \%$ of the health plan operators in the country enforce clinical guidelines. With regard to cardiovascular disease, the reported utilization of guidelines was one of the highest among all diseases analyzed. The percentages for acute myocardial infarction (87\%), congestive heart failure (85\%) and the use of invasive procedures (81.1\%) were higherthan for arterial hypertension (74. $1 \%$ ) and cerebrovascular disease (72.2\%).

CONCLUSION. This study indicated the incipient utilization of clinical guidelines among health plan operators in the Brazilian health system. In general, the cardiovasculararea showed one of the highestrates ofutilization reported in the study. [Rev Assoc Med Bras 2008; 54(5): 400-5]

KEY WORDS: Guidelines. Practice guidelines. Supplemental health. Health benefit plans. Cardiovascular disease. Cardiology.

\section{REFERÊNCIAS}

I. Institute of Medicine. Committee to Advise the Public Health Service on Clinical Practice Guidelines. In: Field MJ, Lohr KN, editors. Clinical Practice Guidelines: Directions for a New Program. Washington (DC): National Academy Press; 1990.

2. Portela, MC. Diretrizes clínicas como instrumento de melhoria da qualidade da assistência suplementar: o papel da Agência Nacional de Saúde Suplementar. In: ANS. Brasil. Ministério da Saúde, organização. Regulação \& saúde: Documentos técnicos de apoio ao Fórum de Saúde Suplementar de 2003. Rio de Janeiro; 2004. v.3, p. I77-210.

3. Bodenheimer T, Wagner EH, Grumbach K. Improving primary care for patients with chronic illness. JAMA. 2002;288: 1775-9.

4. Grimshaw JM, Russell IT. Effect of clinical guidelines on medical practice: a systematic review of rigorous evaluations. Lancet. 1993;342: 1317-22.

5. Grol R, Dalhuijsen J, Thomas S, Veld C, Rutten G, Mokkink H. Attributes of clinical guidelines that influence use of guidelines in general practice: observational study. BMJ. 1998;317:858-61.

6. Grol R. Improving the Quality of Medical Care: building bridges among professional pride, payer profit, and patient satisfaction. JAMA. 200।;286:2578-85.

7. Portela MC, Escosteguy CC, Lima SML, Ferreira VM, Vasconcellos MTL, Brito C. Estudo sobre a utilização de diretrizes clínicas no âmbito da saúde suplementar no Brasil. Relatório Final. Rio de Janeiro: FIOTEC; 2006.

8. McKinlay JB, Link CL, Freund KM, Marceau LD, O'Donnell AB, Lutfey KL. Sources of variation in physician adherence with clinical guidelines: results from a factorial experiment. J Gen Intern Med. 2007; 22:289-96.

9. Caminiti C, Scoditti U, Diodati F, Passalacqua R. How to promote, improve and test adherence to scientific evidence in clinical practice. BMC Health Serv Res. 2005;5:62-73

10. Leite IC, Schramm JMA, Gadelha AMJ, Valente JG, Campos MR, Portela $M C$, et al. Comparação das informações sobre as prevalências de doenças crônicas obtidas pelo suplemento saúde da PNAD/98 e as estimativas pelo estudo Carga de Doença no Brasil. Ciênc Saúde Coletiva 2002;7:733-4I.

1 I. Oliveira GMM, Klein CH e Souza e Silva NA. Mortalidade por doenças cardiovasculares em três estados do Brasil de 1980 a 2002. Rev Panam Salud Publica. 2006; 1 9:85-93.

12. Escosteguy CC, Portela MC, Medronho RA, Vasconcellos MTL, Pharmacological management of acute myocardial infarction in the municipal district of Rio de Janeiro. São Paulo Med J. 200 I ; I 1 9: 193 -9.

13. Bourquin MG, Wietlisbach C, Rickenbach M, Perret F, Paccaud F. Time trends in the treatment of acute myocadial infarction in Switzerland from 1986 to 1993: do they reflect the advances in scientific evidence from clinical trials? J Clin Epidemiol. 1998;51:723-32.

14. McGovern PG, Pankow JS, Shahar E, et al. Recent trends in acute coronary heart disease. Mortality, morbidity, medical care and risk factors. N Engl J Med. 1996;334:884-90.

15. O'Connor GT, Quinton HB, Traven ND, Ramunno LD, Dodds TA, Marciniak TA, et al. Geographic variation in the treatment of acute myocardial infarction: the Cooperative Cardiovascular Project. JAMA. 1999;281:627-33.

16. Pilote L, Califf RM, Sapp S, Miller DP, Mark DB, Weaver WD, et al. Regional variation across the United States in the management of acute myocardial infarction. N Engl J Med. 1995;333:565-72.

17. Venturini F, Romero M, Tognoni G. Patterns of practice for acute myocardial infarction in a population from ten countries. Eur J Clinical Pharmacol. 1999;54:877-86.

18. Peterson ED, Roe MT, Mulgund J, DeLong ER, Lytle BL, Brindis RG, et al. Association between hospital process performance and outcomes among patients with acute coronary syndromes. JAMA. 2006;295:1912-20.

19. Williams SC, Koss RG, Morton DJ, Loeb JM. Performance of top-ranked heart care hospitals on evidence-based process measures. Circulation. 2006; | | 4:558-64.

20. Hedlin D. A procedure for stratification by an extended Ekman rule. J Offic Stat. 2000; 16:15-29.

21. Hájek J. Limiting distribution in simple random sampling from a finite population. Publ Math Inst Hung Acad Sci. 1960;5:361-74.

22. Associação Médica Brasileira e Conselho Federal de Medicina. Projeto Diretrizes. [citado abr 2007]. Disponível em: http:// www. projetodiretrizes.org.br.

23. Sociedade Brasileira de Cardiologia. Diretrizes e Consensos. [citado abr 2007]. Disponível em: http://publicacoes.cardiol.br/consenso. Acesso em abril 2007.

24. Mehta RJ, Montoye CK, Gallogly M, Baker P, Blount A, Faul J, et al. Improving Quality of Care for Acute Myocardial Infarction. The Guidelines Applied in Practice (GAP) Initiative. JAMA. 2002;287: 1 269-76.

25. Ministério da Saúde. Pacto pela Saúde 2006. Portaria no 399/GM de 22/ 02/2006. [citado abr 2007]. Disponível em: http://bvsms.saude.gov.br/ bvs/publicacoes/prtGM399_20060222.pdf.

Artigo recebido: 10/07/07

Aceito para publicação: 18/03/08 